\title{
La problemática táctica, clave en el diseño representativo de tareas desde el enfoque de la pedagogía no lineal aplicadaal deporte \\ Tactical problems, key component of the representative design of tasks within the non-linear pedagogy approach applied to sports \\ *Jaime Serra-Olivares, **Javier Garcia-Rubio \\ *Universidad Católica de Temuco (Chile), **Universidad Autónoma de Chile(Chile)
}

\begin{abstract}
Resumen. La enseñanza de los deportes desde las Perspectivas de los Condicionantes del Aprendizaje Motor y las Aproximaciones Basadas en el Juego y su Comprensión, están ampliamente aceptadas en la actualidad. No obstante, existe la necesidad de un entendimiento superior de los conceptos teóricos que originan el diseño representativo de tareas bajo el enfoque de la Pedagogía No Lineal. En este sentido, el propósito de este trabajo es el de proveer a los profesionales de la Educación Física y el entrenamiento de la estrategia de diseño de tareas basada en la lógica interna de cada categoría deportiva, el Enfoque fundamentado en la Problemática Táctica de los Deportes. Para ello: 1) Se exponen algunos de los principios básicos clave de la psicología ecológica y la teoría de los sistemas dinámicos que han contribuido a la construcción de la pedagogía no lineal; 2) Se presentan los aspectos clave de la perspectiva de los condicionantes del aprendizaje motor y de la dinámica contextual de los deportes; 3) Se exponen los principios pedagógicos de las formas de enseñanza basadas en el juego y su comprensión; 4) Se aborda la relación de los principios de la psicología ecológica y los sistemas dinámicos con el diseño de tareas basado en la problemática táctica. Por último, 5) Se expone un ejemplo en fútbol en relación a las posibilidades de esta novedosa estrategia de diseño.
\end{abstract}

Palabras clave: táctica, enseñanza, tareas, condicionantes, deporte.

Abstract. Nowadays it is widely accepted to teach sports from a motor learning determinant perspective, and a game understanding approach. However, there is a need for a higher understanding of the theoretical concepts that determine the representative design of tasks within the Non-Linear Pedagogy approach. The purpose of this paper is to provide professionals of Physical Education and Sports Coaching with strategies for designing tasks based on the essence of each sport category, as well as an approach focusing on tactical problems in sports. In order to do this, the following procedure was carried out: 1) basic key principles of ecologic psychology and the theory of dynamic systems, which contributed to the construction of non-linear pedagogy, are discussed; 2) the key themes of the motor learning determinants perspective, and of the contextual dynamics of sports, are presented; 3) pedagogical principles of Game-Based Approaches are presented; 4) the relationship between the principles of ecological psychology and dynamic systems theory with task design based on tactical problems is addressed. Finally, 5) An example of this novel design strategy is applied in football .

Keywords: tactical, teaching, tasks, conditions, sport.

\section{Introducción}

Dadas las corrientes teóricas provenientes del constructivismo, los sistemas dinámicos y la psicología ecológica, en las últimas cuatro décadas la enseñanza de los deportes ha sufrido un gran cambio. Se ha progresado de una metodología tradicional orientada al desarrollo de gestos técnicos, a una perspectiva más flexible y adaptable a las necesidades del aprendiz, en la que se le brinda la posibilidad de construir su propio aprendizaje (Gil, Moreno, Claver, Moreno \& del Villar, 2016; Gonzalez-Espinosa, Ibáñez, Feu \& Galatti, 2017). Se trata de un enfoque caracterizado por la pedagogía no lineal y el diseño representativo de tareas. Bajo este prisma, los iniciados son los principales protagonistas, exploran el juego y buscan soluciones (Chow et al., 2006; Tan, Chow, \& Davids, 2012). Este tipo de práctica se aleja de las tareas cerradas/analíticas orientadas a la repetición y reproducción, programando una práctica alternativa, abierta, en la que la herramienta de enseñanza es el juego modificado (Ellis, 1983; Thorpe, Bunker, \& Almond, 1986). El fiel reflejo de este cambio puede apreciarse en la perspectiva de enseñanza centrada en el aprendiz, como las Propuestas de Enseñanza de los Deportes Basadas en el Juego y su Comprensión (GBA) (Bunker \& Thorpe, 1982; Griffin, Mitchell, \& Oslin, 1997; Raab, 2007; Launder, 2001; Mitchell, Oslin, \& Griffin, 2003); y también en aquella centrada en la relación aprendiz-contexto, como la perspectiva de los condicionantes (CLA) del aprendizaje motor (Newell, 1986; Renshaw, Chow, Davids, \& Hammond, 2010).

En esta línea, el estudio de la adquisición de habilidades motrices y el entrenamiento deportivo desde el prisma de los sistemas dinámicos y la psicología ecológica ha alcanzado su apogeo en la última década, complementando ciertos espacios teórico-científicos del constructivismo. Se ha demostrado cómo el marco de la pedagogía no lineal proporciona una sólida base para asegurar la eficacia de la perspectiva de enseñanza centrada en el aprendiz (GBA), y también de la centrada en la relación

Fecha recepción: 18-08-16. Fecha de aceptación: 02-12-16 Javier Garcia Rubio

javiergarciarubio@gmail.com aprendiz-contexto (CLA) (Davids, Button, Araújo, Renshaw, \& Hristovski, 2006; Harvey \& Jarrett, 2014; Tan et al., 2012). Así la utilización de los principios pedagógicos de estas perspectivas continúa en crecimiento, y existe la necesidad de un conocimiento superior, de los conceptos teóricos clave de la pedagogía no lineal. Este conocimiento contribuirá a que los profesionales de la Educación Física y el entrenamiento (PEFyE) desarrollen el enfoque adecuadamente y con autonomía (Castejón Oliva, 2015; Renshaw, Davids, Shuttleworth, \& Chow, 2009). Por estos motivos, este trabajo pretende proveer a los PEFyE de una novedosa estrategia de diseño representativo de tareas basada en la lógica interna de cada categoría deportiva, exponiendo su fundamento teórico-científico en relación a la pedagogía no lineal. Se trata de la estrategia de diseño basada en la Problemática Táctica de los Deportes, cimentada en los principios pedagógicos de las GBA y la CLA.

Sistemas dinámicos, psicología ecológica y pedagogía no lineal

Las teorías de sistemas dinámicos y psicología ecológica permiten comprender la coordinación neurobiológica del movimiento entendiendo al individuo como un sistema dinámico complejo compuesto por varios subsistemas. Estos subsistemas están en continua interacción formando patrones coherentes de comportamiento motriz (Seifert, Button, \& Davids, 2013). Las diferentes configuraciones que pueden adoptar los elementos de tales subsistemas es lo que se conoce como grados de libertad. Éstos, deben ser controlados por el deportista para coordinar el movimiento realizado (Bernstein, 1967; Vickers, 2007). Este proceso se consigue gracias a procedimientos de acoplamiento entre información-movimiento, procesos de degeneración y propiedades como la «multi-estabilidad», «meta-estabilidad» y «variabilidad» de los sistemas frente a los condicionantes del contexto (Mason, 2010; Whitacre \& Bender, 2010).

Puede afirmarse que el deportista y su contexto forman un conjunto que interactúa mecánica e informacionalmente (Araujo \& Davids, 2009). De modo que el rendimiento en el deporte se debe a las adaptaciones intencionales realizadas ante los condicionantes del ambiente, 
impuestos por la realización de una tarea concreta (Araujo, Davids, \& Hristovski, 2006; Newell, 1986). Así, los sistemas dinámicos no lineales, como los deportistas, satisfacen un amplio rango de condicionantes a medida que los comportamientos motrices emergen de la interacción entre los mismos (Travassos, Duarte, Vilar, Davids, \& Araújo, 2012; Travassos, Gonçalves, Marcelino, Monteiro, \& Sampaio, 2014). Por tanto, la adaptación óptima del deportista a cada problema motriz depende de los condicionantes presentes en el contexto concreto de rendimiento, y la realización de una determinada habilidad. Por estos motivos, el interés por comprender los procesos adaptativos en el deporte y su enseñanza-aprendizaje (Renshaw et al., 2009). Esta inquietud es la que ha venido a conformar la corriente teórico-científica de lapedagogía no lineal.

La pedagogía no lineal aplicada al deporte se define como la aplicación de los conceptos y herramientas de las dinámicas no lineales a las prácticas de enseñanza/entrenamiento (Chow et al., 2006). Uno de sus propósitos principales es identificar qué condicionantes afectan a los sistemas dinámicos de movimiento (deportistas), para poder comprender las propiedades emergentes de tales sistemas, y así, planificar procesos pedagógicos en consecuencia (Tan et al., 2012). La pedagogía no lineal requiere, en esencia, del conocimiento de las interacciones entre los condicionantes de las tareas, así como los efectos de su manipulación (Newell, 1986). Esteproceso dotará del conocimientonecesario al PEFyE para contribuir al desarrollo de la capacidad de los deportistas para tomar decisiones y ejecutar acciones conforme a los requisitos del contexto (Chow et al., 2006; Renshaw et al., 2010; Renshaw et al., 2009).

\section{La perspectiva de los condicionantes del aprendizaje motor}

Elrendimiento deportivo depende de las adaptaciones intencionales realizadas por los deportistas ante los condicionantes del ambiente, el contexto y la tarea específica a realizar (Araujo et al., 2006; Davids, Button, \& Bennett, 2008; Kugler, Kelso, \& Turvey, 1982; Seifert, et al., 2014). Estos condicionantes, diferentes en cada situación, han sido denominados limitadores (constraints), y es la interacción entre éstos lo que determina la emergencia de los comportamientos motrices en cada momento (Araujo, et al., 2015; Newell, 1986; Vickers, 2007). Esto se debe a que los condicionantes reducen los grados de libertad del sistema, haciendo improbable el surgimiento de determinadas respuestas motrices (Bernstein, 1967).

Los condicionantes del individuo corresponden a factores cognitivos, afectivos, estructurales..., como: nivel de conocimiento, experiencia, características antropométricas,....Los condicionantes del contexto hacen referencia a factores físicos, como por ejemplo: las características climatológicas, la luminosidad durante la realización de una determinada tarea... Finalmente, los condicionantes de la tarea se refieren a todos los elementos que la rodean, como: reglas de juego, objetivos e intereses técnico-tácticos, número de participantes implicados, etc. En la figura 1 se presenta de manera esquemática la interacción entre tales condicionantes.

Desde esta idea el rendimiento de un sistema dinámico (por ejemplo un jugador de fútbol) para realizar una habilidad concreta como un

\section{Profesor-Entrenador}

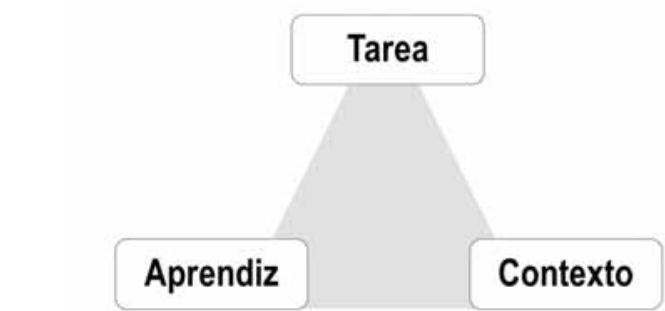

Figura 1. Representación gráfica de los condicionantes que interactúan (del individuo, la tarea y el contexto) durante la emergencia de comportamientos de juego en el deporte. El papel del

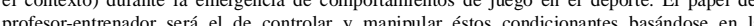
evaluación de las necesidades individuales y del grupo. regate, será diferente en función de los diversos condicionantes. Estos podrían ser si hay mucho viento o poca luz (condicionantes del contexto), si el jugador está cansado por una acción anterior (condicionantes individuales), o si el defensa varía su posición (condicionantes de la tarea). Por tanto, cada acción que realiza un deportista surge de las intenciones e intentos de éste para satisfacer los condicionantes de esa situación concreta (Araujo et al., 2006; Davids et al., 2006; Travassos et al., 2012). Esta visión del deportista como un sistema dinámico complejo, flexible, que se adapta a los contextos de rendimiento cambiantes, ha permitido el estudio de las dinámicas de los condicionantes, y en consecuencia, la fundamentación teórico-científica de los principios pedagógicos de las CLA y GBA (Renshaw et al, 2015; Tan et al., 2012).El estudio de la relación condicionantes del individuo-condicionantes de la tarea ha supuesto un paso más en la fundamentación del diseño representativo de tareas, destacando la importancia de conocer la dinámica contextual del deporte para programar prácticas acordes a las necesidades de los aprendices (Pinder, Davids, Renshaw, \& Araújo, 2011), por ejemplo: en boxeo, vela y baloncesto (Araujo et al., 2006; Davids et al., 2006), o en fútbol (Serra-Olivares, González-Villora, García-López, \& Araújo, 2015; Travassos et al., 2012). Las dinámicas contextuales de los deportes son diferentes, cambiantes y están cargadas de incertidumbre. Por este motivo la necesidad de conocerlas y controlarlas.

La dinámica contextual de los deportes: Los problemas tácticos

El estudio de los comportamientos motrices en el deporte desde la CLA facilita la comprensión de los procesos internos influyentes en la adquisición de habilidades (Aguiar, Botelho, Lago, Maças, \& Sampaio, 2012; Almeida, Ferreira, \& Volossovitch, 2013; Costa et al., 2010). Concretamente, el estudio de los condicionantes de la tarea respecto a la esencia táctica de los deportes, su lógica interna, sus reglas y formas de actuación, ha permitido demostrar que la dinámica contextual de juego está principalmente influenciada por los problemas tácticos inherentes a ese deporte/categoría deportiva, más que por otros condicionantes como el espacio de juego o número de jugadores (Gonzalez-Villora, Garcia-Lopez, Gutierrez-Diaz del Campo, \& Contreras-Jordan, 2010; González Víllora, García López, Vicedo, Carlos, \& Contreras Jordán, 2011; Gutiérrez-Díaz, González-Víllora, García-López, \& Mitchell, 2011; Sanchez-Mora, Garcia Lopez, Del Valle Diaz, \& Solera Martinez, 2011).

Los problemas tácticos determinan la dinámica cambiante de los condicionantes de la tarea respecto a las reglas de acción en ese deporte y los objetivos de los jugadores/equipos implicados (Serra-Olivares et al., 2015). En un partido de baloncesto por ejemplo, en función del contexto táctico, los jugadores atacantes podrán realizar pases, desmarques etc., mientras que los jugadores defensores deberán realizar marcajes, interceptaciones... Igualmente, el jugador atacante con el balón podrá botar, conducir etc., mientras que los jugadores sin balón podrán desmarcarse o hacer un bloqueo. Cada jugador/equipo tiene delimitadas las posibilidades de actuación en función de la categoría deportiva a la que pertenece ese deporte (reglas de acción), el/los roles de juego (en el caso del baloncesto/balonmano/fútbol: atacante con/sin balón o defensor de atacante con/sin balón) o el contexto táctico (ataque, defensa, transiciones) (Gréhaigne \& Godbout, 1995).

Los problemas tácticos se definen como el contexto específico de los problemas motrices a los que se deben enfrentar los jugadores/ equipos (Griffin et al., 1997; Launder, 2001). Estos problemas proporcionan la información necesaria respecto a la incertidumbre del juego, y definen la relación jugador-tarea durante la interacción entre las dinámicas de ambos. Bayer (1986, pp. 52) afirmó que estos problemas «constituyen el punto de partida, la base; representan la fuente de la acción, definen las propiedades invariables sobre las cuales se realizará la estructura fundamental del desarrollo de los acontecimientos». De este modo, se puede considerar la problemática táctica como el conjunto de condicionantes esenciales delatarea que determinan la dinámica contextual de los deportes (grados de libertad), en relación a los componentes decisional y motriz de las habilidades (condicionantes intencionados). En la tabla 1 se presenta como ejemplo la dinámica contextual/proble- 
mática táctica propuesta por Bayer para los juegos de invasión como el fútbol, adaptada en relación a la influencia de los condicionantes del aprendizaje motor sobre los comportamientos de juego.

Tabla 1

Dinámica contextual de los juegos de invasión: Los problemas tácticos de juego

\begin{tabular}{ll}
\multicolumn{1}{c}{ En ataque } & \multicolumn{1}{c}{ En defensa } \\
Conservar la posesión del balón. & Recuperar el balón. \\
Avanzar hacia la meta contraria. & $\begin{array}{l}\text { Impedir la progresión de los jugadores y del balón } \\
\text { Conseguir el objetivo. }\end{array}$ \\
\hline & Proteger la propia meta. \\
\hline
\end{tabular}

Por ejemplo, si se observan las categorías deportivas de deportes de cancha dividida (tenis, padel, bádminton...), o de juegos de invasión (baloncesto, balonmano, fútbol...) planteadas por diversos autores en las GBA, se puede apreciar la similitud de los problemas tácticos entre los deportes de la misma categoría (Almond, 1986; Ellis, 1983). Así, los practicantes de fútbol o baloncesto deberán: conservar la posesión, avanzar hacia la meta contraria y conseguir el objetivo, en la fase de ataque, o proteger la meta, hacerse con la iniciativa y evitar el avance en defensa; igualmente, los practicantes de bádminton o tenis podrán: mantener el móvil en juego, llevar la iniciativa o conseguir el objetivo en ataque, y en defensa deberán: evitar el punto, mantener el móvil en juego o recuperar la iniciativa. Con ello, la esencia táctica de los deportes ha supuesto el eje vertebrador de los principios pedagógicos de las GBA (Griffin et al., 1997; Tan et al., 2012).

Los principios pedagógicos de las GBA y la problemática táctica de los deportes

Los cuatro principios pedagógicos principales de las GBA (muestreo, representación, exageración y complejidad táctica) provienen de la primera aproximación de la enseñanza mediante la comprensión al deporte, la propuesta Teaching Games for Understanding, que fue presentada en su inicio como un modelo de curriculum para el desarrollo de la toma de decisiones y habilidades en los juegos deportivos (Thorpe \& Bunker, 1997; Thorpe et al., 1986, Stolz \& Pill, 2014). En la actualidad, gracias a trabajos como los de Renshaw et al. (2010), Tan et al. (2012) o Renshaw et al. (2015), el estudio de estas propuestas desde el enfoque de la Pedagogía No Lineal y la CLA ha proporcionado un marco teórico relevante respecto al diseño de juegos y tareas representativas del deporte, orientadas al entendimiento del juego, la capacidad para tomar decisiones y la adquisición de habilidades.

El principio pedagógico de muestreo se basa en la enseñanza de los deportes más significativos dentro de cada categoría deportiva. Propone que la selección de los deportes a enseñar debería ofrecer una variedad de experiencias con posibilidades para mostrar las similitudes y diferencias entre los deportes que aparentemente son iguales o diferentes (Thorpe \& Bunker, 1997; Thorpe et al., 1986). Con este propósito, el PEFyE puede ayudarse del sistema de clasificación de los deportes comentado anteriormente (Almond, 1986; Ellis, 1983) para facilitar la integración de deportes con las mismas posibilidades tácticas. De este modo, los aprendices que practican juegos de la misma categoría deportiva tienen la posibilidad de experimentar elementos tácticos similares, así como estrategias de actuación para la consecución de los mismos objetivos. Este proceso contribuye a que sean capaces de identificar la información esencial en relación a la táctica de la categoría deportiva tratada, desarrollen el entendimiento del juego y transfieran sus aprendizajes a otras situaciones similares de enseñanza (García-López, Contreras, Penney, \& Chandler, 2009; Launder, 2001; Raab, 2007).

Por ejemplo, es posible que un niño que desarrolle su capacidad para identificar y ocupar espacios libres para avanzar en juegos de baloncesto, será capaz de desarrollar fácilmente esta capacidad en juegos similares pero en balonmano, en el que las reglas de acción y objetivos tácticos son idénticos: desmarcarse hacia los espacios desocupados para recibir el balón sin oposición y construir situaciones de ataque. Desde el enfoque dela Pedagogía No Lineal y la perspectiva CLA este proceso es explicado por la relación entre las dinámicas de la tarea y las dinámicas intrínsecas del aprendiz (Araujo \& Davids, 2009; Araujo et al., 2006; Travassos et al., 2012). Por este motivo, el diseño representativo de tareas desde este principio debería basarse principalmente en el conocimiento de los condicionantes del individuo y su relación con los condicionantes de la tarea en relación a la esencia táctica. Como puede observarse, el conocimiento de la problemática táctica de los deportes es fundamental para la utilización de este principio pedagógico en el diseño representativo de tareas.

El principio pedagógico de representación, por su parte, se basa en la utilización de tareas reducidas en tamaño de los elementos del juego, representativas de la táctica del deporte/s tratado/s, y que mantengan la misma estructura que la modalidad adulta o de competición. El propósito es modificar el juego original para facilitar la experimentación de situaciones en las desarrollar la conciencia táctica y la toma de decisiones. Se trata de situaciones adaptadas a las necesidades de los aprendices (Mitchell et al., 2003; Raab, 2007; Thorpe et al., 1986). Este principio contribuye al diseño de tareas apropiadas al momento de aprendizaje, proporcionando experiencias en las que se mantienen los acoplamientos entre información-movimiento similares a las que se producen en el juego adulto. De hecho, en salto de trampolín se ha puesto de manifiesto la importancia de la representatividad de las tareas (Barris, Davids \& Farrow, 2013). Un ejemplo es la utilización del Mini-baloncesto como formato de competición en iniciación al baloncesto, en el que no solamente se modifica el tamaño del balón o la altura de la canasta, sino también el número de jugadores y el espacio de juego, facilitando la adaptación táctica de los jugadores a los contextos. De modo que el deber del PEFyE será el de proporcionar situaciones representativas de ese deporte adaptadas a las necesidades del aprendiz, incluida la representatividad táctica (García-López et al., 2009; Gréhaigne \& Godbout, 1995). Se puede apreciar también como el conocimiento y dominio de la problemática táctica por parte del PEFyE será fundamental para el empleo de este principio.

El principio pedagógico de exageración está íntimamente relacionado con el de representación. Se fundamenta en el diseño de tareas representativas del deporte en relación a la problemática táctica que se pretende exploren los participantes. Se subraya el diseño de tareas que faciliten al aprendiz la identificación de las soluciones motrices apropiadas en relación a la problemática táctica concreta. Para ello, desde el enfoque de la pedagogía no lineal el PEFyE deberá manipular los condicionantes de la tarea necesarios para exagerar aquel contenido táctico que pretende desarrollar, proveyendo a los aprendices de un contexto de aprendizaje relevante (ej., en un juego de cancha dividida como tenis o padel, si se reduce el espacio de la pista en anchura y se amplía en profundidad los jugadores deberán desarrollar golpeos como «globos» 0 «dejadas» en lugar de golpeos a los lados del contrario para desestabilizarlo) (Muñoz et al., 2017; Tan et al., 2012).

Si el objetivo fuera el de desarrollar el aprendizaje del pase y el desmarque en los juegos de invasión, se podría orientar el juego hacia el problema táctico de conservar la posesión, principalmente eliminando las metas/porterías. No obstante, debemos tener en cuenta que en los diferentes deportes de las diferentes categorías deportivas los jugadores utilizan comportamientos motrices similares para adaptarse a problemas tácticos distintos (Renshaw et al., 2015; Seifert et al., 2013; Travassos et al., 2014). Por ejemplo, en los juegos de invasión los jugadores no solamente realizan pases y desmarques para conservar la posesión porque están presionados por los contrarios o porque desean temporizar en una acción. Los jugadores también deben hacerlo para avanzar, para realizar un contra-ataque o para construir situaciones de consecución del objetivo. Por tanto, el PEFyE que desee implementar un programa de enseñanza fundamentado en la pedagogía no lineal siguiendo esta propuesta deberá atender a un aspecto elemental; cualquier alteración de los condicionantes de la tarea que afecte a la problemática táctica, afectará también a la manera en la que se desarrollan las interacciones entre las dinámicas del aprendiz y las de la tarea (GutiérrezDíaz et al., 2011; Passos \& Davids, 2015).

El principio pedagógico de complejidad táctica está basado en la utilización de tareas acordes al nivel de aprendizaje y desarrollo de los aprendices. Se trata de tareas en las que los aprendices, a pesar de las 
dificultades propias del proceso, sean capaces de comprender y solucionar los problemas tácticos que se le presentan. En esta línea, si el propósito principal es que comprendan la esencia de los deportes que practican, se deberían elegir para su enseñanza aquellas modalidades/ categorías deportivas con menor complejidad, facilitando el proceso (Tan et al., 2012; Thorpe et al., 1986). Ello implica la progresión en la enseñanza de los deportes utilizando los distintos niveles de complejidad de las diferentes categorías deportivas, e igualmente, los niveles de complejidad dentro de cada categoría deportiva (por ejemplo, se recomienda la iniciación a los deportes de diana previa a la enseñanza de otras categorías deportivas como los deportes de pared/red o campo y bate, debido a la menor complejidad táctica. De igual modo, la progresión de enseñanza en la complejidad táctica dentro de una categoría deportiva implica la adaptación de las tareas a las necesidades de los aprendices, en función de la dificultad de los problemas tácticos planteados: conservar la posesión, avanzar...) (Mitchell et al., 2003).

Diversos estudios han abordado este principio. En el caso de la progresión de enseñanza respecto a la complejidad táctica dentro la cada categoría deportiva de juegos de invasión, por ejemplo, se ha demostrado que los aprendices desarrollan más dificultades para solucionar los problemas propios del contexto táctico de conservación del balón que los relacionados con avanzar o conseguir el objetivo (Sanchez-Mora et al., 2011). Mientras que en estudios en los que se manipularon los condicionantes de la tarea orientando el juego al problema táctico de conservar la posesión, se comprobó una dinámica contextual completamente distinta al juego adulto o de competición(Serra-Olivares, GonzalezVíllora, \& García-López, 2011). Se puede afirmar por tanto que la clave para la utilización adecuada del principio pedagógico de complejidad táctica en el diseño representativo de tareas reside en el profundo conocimiento de la problemática táctica. Esto implica el conocimiento de la influencia de la problemática táctica sobre las dinámicas decisionales y motrices de los practicantes, y más importante, el conocimiento de esa influencia dependiendo de la categoría deportiva.

La filosofía de entrenamiento fundamentada en los principios teóricos básicos de la psicología ecológica, los sistemas dinámicos (la CLA): Relación con la problemática táctica de los deportes

Renshaw et al. (2009), han utilizado hasta doce principios teóricos básicos clave de la psicología ecológica y los sistemas dinámicos para apoyar la filosofía de entrenamiento basada en la CLA. Estos principios apoyan, como veremos, la idea del diseño representativo de tareas fundamentado en la problemática táctica de los deportes. En este sentido, se han mantenido los títulos originales de estos principios en el manuscrito de Renshaw et al. (2009) para facilitar la comprensión de esta relación entre CLA y Problemática Táctica.

La mutualidad entre el deportista y el contexto. Es preciso entender al aprendiz en relación a su contexto. En los deportes colectivos por ejemplo, éste incluye a los compañeros/adversarios, el campo, el/los móviles o implementos... En este sentido, para que un aprendiz sea capaz de desarrollar un comportamiento con precisión, es necesario que detecte las posibilidades (affordances) de ese problema/situación motriz (Tan et al., 2012). Esas posibilidades se refieren a una propiedad del contexto que puede ser detectada como información para facilitar la acción motriz (ej., cuando un compañero está desmarcado proporciona información al jugador con balón de que puede pasarle el balón). Así, las tareas de enseñanza deben ser representativas del contexto/información que pretenden simular (Pinder et al., 2011), incluida su esencia táctica. El diseño de situaciones de enseñanza requiere un amplio conocimiento de los condicionantes específicos del contexto, el individuo y la tarea en cada modalidad deportiva, en relación a su táctica. Es decir, implica el conocimiento de la problemática táctica para poder diseñar tareas representativas que simulen las posibilidades de acción (affordances) del contexto de rendimiento concreto de ese deporte/categoría deportiva (García-López et al., 2009). Por ejemplo, organizar a los jugadores por parejas para realizar pases, uno frente al otro, para desarrollar la habili- dad de pase en balonmano, carece de la riqueza contextual de ese deporte. Por el contrario, simular situaciones en las que los jugadores deben decidir qué tipo de pase utilizar o dónde/cuándo pasar, contribuirá a una mejor relación deportista/contexto (Mitchell et al., 2003; Passos, Araújo, Davids, \& Shuttleworth, 2008). La clave para un diseño adecuado es conocer el momento de aprendizaje y manipular los condicionantes necesarios para proporcionar situaciones relevantes respecto a la problemática táctica tratada.

Percepción y acción están acopladas. Este principio se fundamenta en que la información guía al movimiento, aunque el hecho de moverse influye en qué cantidad/tipo de información puede ser utilizada por los aprendices. La base, es el diseño de tareas que proporcionen el contexto de aprendizaje óptimo en el que los aprendices puedan percibir la información esencial respecto a ese problema motriz, para desarrollar comportamientos funcionales acordes al mismo. Por ejemplo, si se pretendiese desarrollar la habilidad de «toque de dedos» en voleibol, no tendría sentido desarrollarla principalmente mediante pases a una pared. Este proceso carece de la información perceptiva relevante respecto a la habilidad, a diferencia de la realización del gesto frente a un compañero y/o la red (Brunswik, 1956; Carvalho, Iglesias, Araújo, \& García González, 2011).

En relación a lo anterior, algunos trabajos como los de SanchezMora et al. (2011) o Serra-Olivares, Gonzalez-Víllora, et al. (2011) han observado en el análisis de los juegos de invasión, que la dinámica decisional de los pases y desmarques es diferente en función del problema táctico al que se enfrentan los jugadores. Estos autores comprobaron que determinados contextos tácticos requerían de la adaptación específica de los jugadores a los mismos, demostrando la diferencia entre la información recibida por los jugadores en unos contextos y en otros. De manera que la información proporcionada a los practicantes no es similar en una tarea en la que se exagera el problema táctico de conservar la posesión en un juego de invasión y se eliminan las metas, por ejemplo, a la información proporcionada en un juego en el que se mantienen. En el primero de los juegos los jugadores atacantes solamente pueden conservar el balón, mientras que en el segundo también pueden avanzar o conseguir el objetivo. Ello implica que la manipulación de la problemática táctica en una tarea influye sobre la información proporcionada a los aprendices en la misma. Por tanto, el principio «percepción y acción están acoplados» también apoya el diseño representativo de tareas basado en la problemática táctica, debido a que cualquier alteración de la dinámica contextual afectará a la información relevante proporcionada a los jugadores (Kugler et al., 1982).

La auto-organización ante los condicionantes: El rendimiento emerge como consecuencia de la interacción entre los condicionantes del individuo y los del equipo. Auto-organización frente a los condicionantes. Este principio subraya que los aprendices desarrollan los comportamientos por medio de procesos de auto-organización, producidos por la interacción entre los condicionantes del individuo, la tarea y el contexto (Araujo et al., 2006). Si la forma de los condicionantes es estable, la respuesta ante esos determinados condicionantes puede ser similar. Por el contrario, si las dinámicas de los condicionantes cambia, el individuo deberá adaptarse a los cambios mediante procesos de re-organización (Davids et al., 2006; Davids et al., 2008). Por ello, la importancia de proporcionar situaciones diferentes en cuanto a las dinámicas de los condicionantes, para facilitar el desarrollo de la capacidad para flexibilizar los comportamientos y adaptarse a los cambios impuestos, principalmente por la tarea y el contexto. Este proceso implica el conocimiento sobre cómo influyen determinados condicionantes clave y su forma respecto al resultado de las respuestas. Otra vez, el conocimiento de la problemática táctica se sugiere fundamental.

Por ejemplo, Gutiérrez-Díaz et al. (2011) observaron cómo la interacción entre los condicionantes individuales (edad y nivel de experiencia), con los de la tarea (los problemas tácticos), daba lugar a niveles de rendimiento de juego diferentes. El PEFyE que pretenda implementar un programa fundamentado en la pedagogía no lineal atendiendo al principio de auto-organización, deberá entender por tanto la continua influencia de la problemática táctica sobre el rol adaptativo y funcional 
del movimiento, ante las demandas de la determinada tarea y contexto (Araujo et al., 2006; Davids et al., 2006; Seifert et al., 2013).

El desarrollo de rendimiento es un proceso no lineal. Las nuevas corrientes de estudio han demostrado que el desarrollo del talento no es lineal y sí discontinuo. Se trata de un proceso multidimensional afectado por la interacción entre los componentes del deporte en cuestión que limitan el rendimiento. En esta línea, la tardía emergencia de alguno de los factores individuales del aprendiz (a nivel físico, madurativo...) actúa como un limitador del rendimiento a obtener, dado que los niños crecen a diferentes ritmos y desarrollan también diferentes ritmos de aprendizaje (ej., corrientes teóricas actuales sobre el efecto de la edad relativa). Por ello, las recomendaciones de la pedagogía no lineal se relacionan con la alteración del equipamiento utilizado con los aprendices en los contextos de práctica, y el análisis de cómo afecta la exposición de los aprendices a nuevos contextos (Renshaw et al., 2009). De modo que el rendimiento es cambiante y diferente en función del momento de aprendizaje y la interacción con el contexto (Renshaw et al., 2015). Este principio de que «el rendimiento no es lineal» implica por tanto el conocimiento de la problemática táctica, para poder adaptar la complejidad de las tareas representativas utilizadas a las necesidades de los aprendices.

La variabilidad es esencial para el desarrollo del rendimiento. Las corrientes teóricas de la psicología ecológica y los sistemas dinámicos permiten afirmar que el deportista es un sistema de movimiento que se adapta de manera flexible a los cambios del entorno. Por este motivo, se insta a la utilización de tareas variadas en las que desarrollar las propiedades de «meta-estabilidad», «variabilidad» y flexibilidad», entre otras (Renshaw et al., 2009; Seifert et al., 2013). En este sentido, el diseño representativo de tareas de enseñanza basado en la problemática táctica ofrece una amplia gama de posibilidades a la hora deplantear situaciones acordes a los objetivos de la CLA, las GBA, y las necesidades de los principiantes (García-López et al., 2009; Harvey \& Jarrett, 2014; Renshaw et al., 2015). Esto puede apreciarse por ejemplo cuando un PEFyE utiliza variantes de una tarea y adaptaciones de los condicionantes, para facilitar que los aprendices logren el objetivo de la tarea mediante diferentes comportamientos adaptados y funcionales al contexto (repetición sin repetición) (Bernstein, 1967).

El individuo es el foco. Una de las bases de la pedagogía no lineal sostiene la formación del deportista/equipo/grupo utilizando a éste como el centro del proceso de enseñanza-aprendizaje. Para ello, se propone atender a las características del mismo (capacidades, limitaciones, etc.), con el objetivo de contribuir en el desarrollo de habilidades. Entender las dinámicas intrínsecas como un sistema dinámico facilitará el desarrollo de aprendizajes significativos (ej., un entrenador debe conocer las características de su equipo y de la competición en la que participa, para adaptar sus actividades a las necesidades de ese equipo en cada momento) (Newell, 1986; Renshaw et al., 2010). Por este motivo, si uno de los objetivos principales de la CLA es asegurar el desarrollo de acoplamientos entre información-movimiento, y el objetivo de las GBA es el de desarrollar la capacidades de toma de decisión y entendimiento del juego de los aprendices, parece lógico fundamentar el diseño representativo de tareas en la problemática táctica de los deportes. Por ejemplo, en deportes de cancha dividida, una vez que el jugador es capaz de mantener el móvil en juego, el siguiente paso sería desarrollar la capacidad para llevar la iniciativa e intentar conseguir punto. El PEFyE debe conocer por tanto las limitaciones de los aprendices para golpear la pelota, y también aquellas limitaciones relacionadas con la comprensión del juego y la problemática táctica, para poder adaptar el proceso de enseñanza (Carvalho et al., 2011; Renshaw et al., 2015).

El equipo es un sistema dinámico abierto. La dinámica contextual de los deportes se ve influenciada por los problemas tácticos inherentes a estos (Gonzalez-Villora et al., 2010; Gutiérrez-Díaz et al., 2011; Sanchez-Mora et al., 2011). Los condicionantes de los deportes de equipo determinan la emergencia de los comportamientos motrices individuales, de acuerdo a la dinámica contextual concreta de ese deporte/categoría deportiva (Araújo, Davids, Bennett, Button, \& Chapman, 2004; García-Rubio, Gómez, Cañadas, \& Ibáñez, 2015; Travassos et al., 2014). Esto quiere decir que las conductas de juego se forman y emergen desde la interacción entre las dinámicas individuales dentro de las dinámicas del/los equipos (Almeida et al., 2013; Costa et al., 2010). Por este motivo se recomienda la utilización de tareas representativas en las que no se descontextualice la problemática táctica. Este procedimiento facilitará los procesos de «auto-organización» ante situaciones de juego con similitud táctica, aunque diferentes y cambiantes («repetición sin repetición») respecto a la práctica (Passos et al., 2008). Por ejemplo, ubicar a un grupo de futbolistas en filas para realizar situaciones de 3 contra el portero y realizar una secuencia de pases y remate representa una situación repetitiva y descontextualizada. Por el contrario, resultaría más interesante proponer situaciones de contra-ataque 3 contra 3 donde los defensas tuvieran movimientos limitados o existieran áreas que no pudieran ocupar. Estos condicionantes facilitarían la «repetición sin repetición» de comportamientos de juego, y la adaptación individual de los jugadores a los movimientos del equipo. Se aprecia así, cómo este principio teórico también apoya la propuesta de diseño representativo basado en la problemática táctica, que podría favorecer la planificación de los procesos de enseñanza-aprendizaje a edades tempranas (Serra-Olivares, García-López, \& Sánchez-Mora, 2011; SerraOlivares, Gonzalez-Víllora, et al., 2011).

El entrenamiento es el equilibrio entre mantener la estabilidad vs. proporcionar inestabilidad. Fundamentada en los principios de la teoría de los sistemas dinámicos que sustentan la idea de que la inestabilidad de los sistemas facilita el desarrollo de la propiedad de re-organización, se propone el planteamiento de tareas abiertas, cambiantes y variadas, que contribuyan a que el aprendiz progrese en su aprendizaje (Tan et al., 2012). La clave es diseñar tareas que estimulen el proceso de transición del sistema (el deportista) desde un estado/os a otro/os de aprendizaje (Renshaw et al., 2010). En esta línea, un entrenador de baloncesto podría utilizar frecuentemente tareas de conservación del balón eliminando las canastas y reduciendo el número de jugadores para facilitar el aprendizaje del pase y el desmarque hacia el espacio libre. No obstante, la utilización de este tipo de tareas podría suponer situaciones de inestabilidad en un momento del aprendizaje concreto, no así en otros. Los jugadores sin balón por ejemplo entenderán pasado un tiempo que es importante ocupar cualquier espacio libre de marca para recibir un pase, pero... ¿ ¿serán capaces de ocupar el espacio libre adecuado en un juego en el que sí haya canastas?

Si bien es cierto que la variabilidad e inestabilidad de las tareas facilitan los procesos de auto-organización y re-organización de los sistemas (aprendices) contribuyendo al desarrollo de las capacidades de flexibilidad y adaptabilidad (Seifert et al., 2013), el PEFyE debe comprender que la problemática táctica determina la dinámica contextual de los condicionantes de la tarea (Gutiérrez-Díaz et al., 2011; SanchezMora et al., 2011). Ello implica que las tareas de enseñanza deben ser abiertas, variadas e inestables, facilitando escenarios cambiantes (ej., modificando espacios, número de jugadores, tiempos de juego...), pero también implica comprender tal inestabilidad y qué transferencia tendrán esas tareas sobre el resultado final del aprendizaje deseado (GarcíaLópez et al., 2009). Este hecho pone de manifiesto la importancia de conocer la dinámica contextual (los problemas tácticos) y la interacción de ésta con las dinámicas intrínsecas de los aprendices (Araujo \& Davids, 2009).

Movimientos co-adaptativos. Implicaciones para la práctica. Los deportistas se adaptan, como sistemas dinámicos, a la dinámica contextual de los deportes. En esta línea, se afirma que los comportamientos emergentes durante el juego se desarrollan por las interacciones entre los componentes de este sistema, las cuales conllevan procesos de coadaptación a los condicionantes. Desde el enfoque de la pedagogía no lineal se defiende el concepto de co-adaptación en el aprendizaje, instando a la no separación, por ejemplo, entre atacante/defensor, con el objetivo de que las dinámicas de ambos sistemas (deportistas) conlleven a la emergencia de los comportamientos deseados por el PEFyE. No obstante, el PEFyE debe comprender que no existen decisiones perfectas durante el juego que puedan predecirse con anterioridad, pues el rendimiento conlleva procesos de adaptación a los condicionantes 
concretos en cada momento (García-Rubio et al., 2015). Así pues, los PEFyE deben evitar el diseño de tareas que limiten los procesos de toma de decisión y acción de los deportistas. Por el contrario, se recomienda la utilización de tareas representativas que conlleven condicionantes primarios, por ejemplo, distancia entre los jugadores, dimensiones de juego, etc., y condicionantes secundarios, comprendiendo que la interacción entre las acciones de los deportistas conllevan la emergencia de otros condicionantes y comportamientos emergentes (Renshaw et al., 2009).

En relación a lo anterior la propuesta de diseño representativo fundamentado en la problemática táctica, se presenta como una estrategia adecuada para la planificación de situaciones de enseñanza que conlleven la emergencia de comportamientos flexibles y adaptables (Araújo et al., 2004). Por ejemplo, algunos autores como Gutiérrez-Díaz et al. (2011) o Serra-Olivares et al. (2015) han apreciado cómo los jóvenes que se inician al deporte deciden de manera adaptable al contexto táctico que se les presenta, es decir, co-adaptan sus comportamientos en cada momento a los intereses individuales/grupales en contraposición a los intereses individuales/grupales del adversario/os.

Fomentar la creatividad en el aprendizaje y el rendimiento. Las nuevas corrientes explicativas del rendimiento en el deporte permiten demostrar las propiedades de adaptabilidad y flexibilidad de los deportistas ante los condicionantes impuestos por el contexto y la tarea (Seifert et al., 2013). Así, la pedagogía no lineal promueve la planificación de tareas representativas de la situación/es de rendimiento que conlleven la emergencia de comportamientos funcionales a los condicionantes impuestos, permitiendo la exploración y descubrimiento de las posibilidades motrices.

La aproximación al aprendizaje y el rendimiento desde esta perspectiva invita la utilización de tareas abiertas y juegos modificados que supongan un desafío a los practicantes. Estos juegos contribuirán no solamente al gusto por la práctica, sino también a la consecución de mayores niveles técnicos, tácticos o físicos (Serra-Olivares, GarcíaLópez, et al., 2011). En este sentido, ha sido demostrado que con la práctica de situaciones tácticamente similares del juego adulto o competición, los jóvenes pueden enfrentarse a situaciones simuladas en las que explorar sus posibilidades y dificultades (Memmert, 2010; Memmert \& Roth, 2007). Estos hallazgos también apoyan el diseño de tareas fundamentado en la problemática táctica, en donde los iniciados puedan desarrollar comportamientos tácticos creativos y eficientes.

Quéentendemos por aprendizaje natural, ¿aprendizaje implícito? Actualmente, puede afirmarse que el aprendizaje motor no es un proceso que pueda beneficiarse únicamente de instrucciones verbales de manera explícita y un incremento en del feedback (Brunswik, 1956; Davids et al., 2008). Desde la perspectiva de la pedagogía no lineal se aboga por un tipo de aprendizaje implícito caracterizado por la utilización de tareas en las que los aprendices puedan descubrir por sí solos las soluciones a los problemas planteados, sin necesidad de estar constantemente proporcionando instrucciones. Este tipo de práctica puede apreciarse bajo el prisma de la CLA (Chow et al., 2006; Davids et al., 2008), y también en las GBA(Bunker \& Thorpe, 1982; Tan et al., 2012). Se ha observado que mediante el diseño representativo de tareas basadas en la problemática táctica de los deportes se puede proporcionar a los aprendices situaciones en las que sean partícipes de su aprendizaje, explorando/descubriendo posibles soluciones y comprendiendo la lógica interna del juego. Todo ello sin necesidad de recibir feedback de ningún tipo por parte del PEFyE (Sanchez-Mora et al., 2011).

¿Práctica cerrada o Práctica abierta?: Una explicación ecológica. Si bien es cierto que la cantidad de práctica realizada por el deportista se ha relacionado con sunivel de rendimiento, también lo es el hecho de que con el tiempo, la calidad de esa práctica ha cobrado mayor importancia en la comunidad científica y en la práctica profesional. Este aspecto está sustentado en el concepto del efecto de interferencia, que sugiere que si bien la utilización de tareas cerradas es efectiva en la primera fase de práctica, no favorece la retención y la trasferencia a largo plazo, a diferencia de las tareas abiertas (Brady, 1998). Se ha demostrado que mediante la utilización de GBA se consiguen alcanzar niveles similares e incluso superiores de conocimiento y habilidad a aquellos alcanzados con prácticas tradicionales (Harvey \& Jarrett, 2014). Gracias a la práctica de situaciones tácticas similares se pueden lograr altos niveles de transferencia de aprendizajes de unas situaciones a otras. Se considera una metodología motivante, innovadora y diferente a las anteriores, la cual permite un gran nivel de participación e involucración por parte del alumnado (García-López et al., 2009). Se trata por tanto de un tipo de práctica en la cual los jugadores, en interacción con los compañeros, adversarios y el contexto, podrían desarrollar comportamientos flexibles y adaptables a las situaciones de rendimiento cambiantes. Este aspecto se ve reforzado por los hallazgos, observados desde la CLA y el enfoque de la pedagogía no lineal (Davids et al., 2006; David Davids, Araújo, Button \& Renshaw, 2007; Davids et al., 2008).

Con todo lo anterior, a continuación se expone un ejemplo en la modalidad de un juego de invasión como es el fútbol, en relación a las posibilidades de la estrategia de diseño de tareas de enseñanza fundamentada en la problemática táctica de los deportes. Ello, con el propósito de proveer a los PEFyE de una estrategia novedosa y de calidad, para el desarrollo de la comprensión del juego y la adquisición de habilidades en el deporte.

La problemática táctica como elemento clave del diseño representativo de tareas de enseñanza: Un ejemplo en fútbol joven

Los hallazgos de varias investigaciones realizadas en torno a la comprensión y entendimiento de los jóvenes deportistas a la problemática táctica, permiten afirmar que: 1) La dificultad para tomar decisiones y ejecutar acciones está influenciada por el número y tipo de problemas tácticos de las situaciones de juego; 2) Existe un desconocimiento total de los problemas tácticos por parte de los jóvenes, tanto en ataque como en defensa; 3) Los aprendices poseen limitaciones y dificultades en el conocimiento de varios elementos técnico-tácticos individuales y también grupales; 4) Se poseen conocimientos más centrados en la técnica que en las estrategias acción a emplear; 5) Se posee un conocimiento poco desarrollado sobre los procedimientos de acción; 6) Existe una fuerte relación entre lo que los aprendices saben y como lo aplican (Gonzalez-Villora et al., 2010; Serra-Olivares, Gonzalez-Víllora, et al., 2011; Serra-Olivares et al., 2015), Este panorama demuestra las carencias respecto al entendimiento de la lógica interna de juego, indicando la necesidad de adoptar una concepción diferente del proceso de enseñanza, incluido el diseño representativo de tareas.

En este sentido, el diseño de tareas fundamentado en la problemática táctica se presenta como una estrategia imprescindible para el buen desarrollo de la práctica profesional bajo el prisma de la pedagogía no lineal (Serra-Olivares et al., 2015; Tan et al., 2012). El profesional encargado de la formación de jóvenes deportistas debe recordar que todas las acciones de juego poseen una dificultad diferente y por tanto una calidad distinta en función de la dinámica contextual táctica del problema motriz planteado (Araujo \& Davids, 2009). La dificultad para desarrollar acciones está no solamente influenciada por el número de jugadores, el espacio, el tamaño o el peso del móvil. Los deportistas deciden y ejecutan también dependiendo de la problemática táctica del contexto en el que desarrollan sus habilidades La complejidad táctica de las acciones deportivas se ve afectada por los intereses y objetivos propios y del adversario/os en cada momento (Serra-Olivares et al., 2015).

En este sentido, es necesario comprender que cualquier estrategia de modificación, sobre todo si influye a la problemática táctica, afectará de un modo u otro a: (a) Las reglas de acción a emplear; (b) Las habilidades técnico-tácticas a las que recurrir y; (c) Las soluciones motrices que desarrollar. En definitiva, el diseño de tareas influirá en la manera en la que los aprendices interactúan con el juego y el contexto y desarrollan su aprendizaje.

Ejemplo de diseño representativo de una tarea de enseñanza en fútbol fundamentado en la problemática táctica de este deporte/categoría deportiva

Supongamos que el objetivo de un PEFyE encargado de la forma- 
ción de un grupo de jóvenes deportistas de10-12 años, fuera el de introducir a sus pupilos a los juegos de invasión como el baloncesto, el balonmano o el fútbol, concretamente, a los conocimientos y habilidades propias de éste último (fútbol). La organización de los contenidos de iniciación a este/os deportes consistirá, en la identificación de la problemática táctica de la categoría deportiva. Así, el profesional tendrá presente que en este tipo de deportes los jugadores colaboran en ataque para: mantener la posesión, avanzar o conseguir el objetivo; y en defensa, colaboran para hacerse con la iniciativa, evitar el avance y proteger la propia meta (Bayer, 1986). Posteriormente, en el PEFyE seleccionará de forma específica las habilidades a desarrollar por sus pupilos atendiendo a la problemática táctica identificada y a las premisas básicas de la pedagogía no lineal. El profesional deberá reflexionar aquí sobre los problemas tácticos en los que se desarrollan las habilidades específicas seleccionadas, así como los principios pedagógicos de las GBA que empleará: muestreo, representación, exageración y/o complejidad táctica, en función del objetivo didáctico. Todo este proceso deberá asegurar además, que las estrategias utilizadas se adecúan a la filosofía de entrenamiento basada en la pedagogía no lineal.

Con ello, el proceso de diseño representativo de tareas fundamentado en la problemática táctica se sintetiza en cinco fases: 1) Identificar la problemática táctica del deporte/categoría deportiva a enseñar; 2) Seleccionar el/los principios tácticos sobre los que construir la tarea/as y las habilidades concretas a desarrollar por los aprendices; 3) Seleccionar el/los principios pedagógicos más adecuados para construir y utilizar la tarea; 4) Diseñar e implementar la tarea atendiendo a las características madurativas y nivel del aprendiz/grupo; 5) Comprobar la adecuación de la tarea a la filosofía de entrenamiento de la pedagogía no lineal y a las necesidades de aprendizaje concretas del aprendiz/grupo; 6) Etapa final de cuestionamiento sobre el efecto de la tarea sobre el objetivo didáctico, y en su caso, re-diseño, adaptación o utilización de una nueva tarea y proceso.

En la figura 2 se presenta una tarea representativa de enseñanza del juego de invasión de fútbol para un grupo de nivel medio de pericia y 10 12 años de edad. El propósito principal es el de desarrollar las habilidades de pase y desmarque en contextos de conservación y avance en situaciones de ataque. Para ello, se han utilizado los principios pedagógicos de representación y exageración de las GBA de la pedagogía no lineal para simular los contextos tácticos seleccionados para este deporte/categoría deportiva. Igualmente, el número de jugadores y el espacio de juego han sido adaptados de acuerdo a las propuestas de algunos expertos en la temática (Memmert, 2010; Memmert \& Roth, 2007), y los hallazgos de varios estudios sobre estas variables (Aguiar et al., 2012; Serra-Olivares et al., 2015).

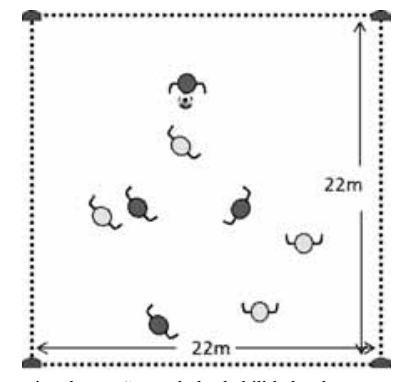

Figura 2. Tarea representativa de enseñanza de las habilidades de pase y desmarque en contextos de conservación y avance de juegos de invasión (fútbol). El juego "Pies quietos" consiste en un de conservación y avance de juegos de invasión (fútbol). El juego "Pies quietos consiste en un entrenta

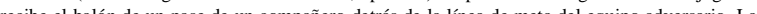

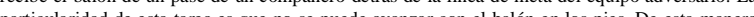

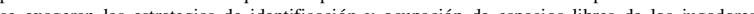
se exage las esta

Como se puede observar en la figura 2, se han utilizado los principios pedagógicos de representación y exageración reduciendo el espacio de juego y el número de jugadores, eliminando las porterías y alterando las formas de actuación y puntuación, para facilitar la experimentación deliberada de situaciones en las que desarrollar la conciencia táctica y la toma de decisiones. De este modo se provee al aprendiz de contextos de aprendizaje relevantes que mantienen la misma estructura que la moda- lidad adulta o de competición, contribuyendo así a la identificación de soluciones apropiadas en relación a la problemática táctica(Serra-Olivares et al., 2015; Tan et al., 2012). Así, los jóvenes pueden construir sus respuestas y coordinar los grados de libertad de movimiento desarrollando propiedades propias de los sistemas dinámicos como la «multiestabilidad», «meta-estabilidad» y «variabilidad» (Bernstein, 1967; Vickers, 2007).

Además, se puede apreciar que el tipo de tarea planteada en cuestión se adecúa a la filosofía de entrenamiento basada en las teorías de la psicología ecológica y los sistemas dinámicos. En la misma se mantiene la mutualidad entre el deportista y el contexto, y las habilidades de percepción y acción están acopladas, pues la problemática táctica implícita lo permite. Igualmente, el contexto táctico de la tarea conllevará la auto-organización de los participantes a los condicionantes del individuo y el equipo, permitiendo el desarrollo del rendimiento de forma lineal, adaptándose a sus características/necesidades en función de la edad/nivel ejemplificado. Por otro lado, la naturaleza táctica de la tarea es variable, aspecto que favorecerá el desarrollo óptimo de la pericia en los dominios de aprendizaje planteados (relación pasador-receptor), ubicando al aprendiz como el foco del proceso. De igual modo, la dinámica táctica de la tarea conllevarála asunción y desarrollo de objetivos/intereses individuales acordes a los propios de cada equipo, resultando en un contexto de entrenamiento caracterizado por la estabilidad/inestabilidad de las situaciones de juego. Esto facilita también la emergencia de respuestas creativas y movimientos co-adaptativos, y fomenta las el aprendizaje implícito. Este hecho se debe a que se trata de una tarea abierta en las que se decide y ejecuta libremente de manera funcional.

\section{Conclusión}

En definitiva, el diseño representativo de tareas de enseñanza fundamentado en la problemática táctica se presenta como una estrategia ideal, efectiva y fácilmente aplicable en los diferentes ámbitos educativos: escolar, de entrenamiento, de ocioy recreación. Puede ser extrapolado a cualquier categoría deportiva y deporte concreto. Su utilización permite el abordaje adecuado de los procesos de iniciación deportiva facilitando contextos de práctica motivantes y significativos, adaptados a las características y necesidades de los aprendices. Por todo lo anterior, invitamos a su utilización como medio efectivo e innovador de enseñanza propio de las GBA.

\section{Aplicaciones prácticas}

Al igual que en otros estudios, se ha encontrado que hay disparidad en el conocimiento que los investigadores generan y cómo los entrenadores utilizan este conocimiento (Stolz \& Pilz, 2014). Este trabajo no pretende si no dotar a los PEFyE de unos principios que aplicar para generar mejores experiencias de aprendizaje a los alumnos y jugadores. Se ha demostrado que la limitación del espacio de juego, espacios de juego donde los jugadores pueden moverse libremente o espacios que los jugadores no pueden abandonar, afecta tanto a las variables tácticas como a las físicas y fisiológicas (Gonçalves, Esteves, Folgado, Ric, Torrents \& Sampaio, 2016). Los PEFyE deben utilizar esta información para enriquecer y mejorar sus sesiones de entrenamiento o enseñanza, de acuerdo a las necesidades de los alumnos y jugadores. Por ejemplo, en un equipo de fútbol, durante la pretemporada, si se necesita que los jugadores mejoren su condición física, se utilizarán situaciones donde estos puedan moverse libremente por el espacio. Por otra parte, si lo que se busca es dificultar la coordinación táctica de los jugadores, se utilizarán espacios de juego limitados, donde los jugadores deberán probar nuevas tomas de decisión para solucionar los problemas de juego.

\section{Futuras líneas de investigación}

Hasta donde sabemos, una de las carencias de los modelos de pedagogía no lineal son investigaciones empíricas sobre los efectos de 
las mismas en grupos homogéneos en deportes colectivos. Dentro de la enseñanza deportiva, la comprobación de estos efectos debe hacerse mediante la validación de unidades didácticas de deportes colectivos y su puesta en práctica. La creación de estas unidades didácticas sobre un mismo deporte y los mismos contenidos bajo una aproximación no lineal y otra técnica o mecanicista, que sean validadas por expertos, su posterior aplicación y evaluación de los aprendizajes de los alumnos a través de herramientas de evaluación diseñadas a tal efecto, se considera de urgente necesidad para comparar los aprendizajes bajo diferentes metodologías/aproximaciones.

\section{Referencias}

Aguiar, M., Botelho, G., Lago, C., Maças, V., \& Sampaio, J. (2012). A review on the effects of soccer small-sided games. Journal of Human Kinetics, 33, 103-113.

Almeida, C. H., Ferreira, A. P., \& Volossovitch, A. (2013). Offensive sequences in youth soccer: effects of experience and small-sided games. Journal of Human Kinetics, 36(1), 97-106.

Almond, L. (1986). Reflecting on themes: A games classification. Rethinking Games Teaching, 71-72.

Araujo, D., \& Davids, K. (2009). Ecological approaches to cognition and action in sport and exercise: Ask not only what you do, but where you do it. International Journal of Sport Psychology, 40(1),

Araújo, D., Davids, K., Bennett, S. J., Button, C., \& Chapman, G (2004). Emergence of sport skills under constraints. In Williams,A. M., \& Hodges, N. J. (Eds.). Skill acquisition in sport: Research, theory and practice. London: Routledge.

Araújo, D., Davids, K., Diniz, A., Rocha, L., Santos, J. C., Dias, G., \& Fernandes, O. (2015). Ecological dynamics of continuous and categorical decision-making: The regatta start in sailing. European Journal of Sport Science, 15(3), 195-202.

Araujo, D., Davids, K., \& Hristovski, R.(2006). The ecological dynamics of decision making in sport. Psychology of Sport and Exercise, 7(6), 653-676.

Barris, S., Davids, K., \& Farrow, D. (2013). Representative learning design in springboard diving: Is dry-land training representative of a pool dive? European Journal of Sport Science, 13(6), 638-45.

Bayer, C. (1986). La Enseñanza de los juegos deportivos colectivos: baloncesto, futbol, balonmano, hockey sobre hierba y sobre hielo, rugby, balonbolea, waterpolo. Barcelona: Hispano europea.

Brady, F. (1998). A theoretical and empirical review of the contextual interference effect

and the learning of motor skills. Quest, 50, 211-223.

Bernstein, N.(1967). The control and regulation of movements. London: Pergamon Press.

Brunswik, E. (1956). Perception and Ihe representative design of psychology experiments. Berkeley: California: University of California Press.

Bunker, D., \& Thorpe, R. (1982). Amodel for the teaching of games in secondary schools. Bulletin of Physical Education, 18(1), 5-8.

Carvalho, J., Iglesias, D., Araújo, D., \& García González, L. (2011). El entrenamiento de la toma de decisiones en el tenis:¿qué fundamentos científicos se pueden aplicar en los programas de entrenamiento? Revista de Psicología del Deporte, 20, 767-783.

Castejón Oliva, F.J. (2015). La investigación en iniciación deportiva válida para el profesorado de educación física en ejercicio. Retos, (28), 263-26

Costa, I., Garganta, J., Greco, P., Mesquita, I., Silva, B., Müller, E., Castelao, D., Revelo, A., \& Seabra, A. (2010). Analysis of tactical behaviours in small-sided soccer games: Comparative study between goalposts of society soccer and Futsal. The Open Sports Sciences journal, 3, 10-12.

Chow, J. Y., Davids, K., Button, C., Shuttleworth, R., Renshaw, I., \& Araujo, D. (2006). Nonlinear pedagogy: a constraints-led framework for understanding emergence of game play and movement skills.
Nonlinear Dynamics, Psychology, and Life Sciences, 10(1), 71103.

Davids, K., Button, C., Araújo, D., Renshaw, I., \& Hristovski, R. (2006). Movement models from sports provide representative task constraints for studying adaptive behavior in human movement systems. Adaptive Behavior, 14(1), 73-95.

Davids, K., Button, C., \& Bennett, S. (2008). Dynamics of skill acquisition: A constraints-led approach. Champaing: Human Kinetics.

Davids, K., Araújo, D., Button, C., \& Renshaw, I. (2007). Degenerate brains, indeterminate behaviour; and representative tasks. In G. Tenenbaum \& R. C. Eklund(Eds.), Handbookof sport psychology (Third ed.). London: John Wiley \& Sons.

Ellis, M. (1983). Similarities and differences in games: A system for classification. Paper presented at the Congreso Mundial AIESEP, Roma.

García-López, L. M., Contreras, O. R., Penney, D., \& Chandler, T. (2009). The role of transfer in games teaching: Implications for the development of the sports curriculum. European Physical Education Review, 15(1), 47-63.

García-Rubio, J., Gómez, M. Á., Cañadas, M., \& Ibáñez, S. J. (2015). Offensive Rating-Time coordination dynamics in basketball. Complex systems theory applied to Basketball. International Journal of Performance Analysis in Sport, 15(2), 513-526.

Gil, A., Moreno, P., Claver, F., Moreno, A., \& del Villar, F. (2016). Manipulación de los condicionantes de la tarea en Educación Física: Una propuesta desde la pedagogía no lineal. Retos, (29), 22-27.

Gonçalves, B., Esteves, P., Folgado, H., Ric, A., Torrents, C., \& Sampaio, J. (2016). Effects of pitch area-restrictions on tactical behavior, physical and physiological performances in soccer large-sided games. The Journal of Strength \& Conditioning Research.Gonzalez-Villora, S., Garcia-Lopez, L.M., Gutierrez-Diaz del Campo, D., \& Contreras-Jordan, O.R. (2010). Tactical awareness and decision making in youth football players (12 years): A descriptive study. Infancia y Aprendizaje, 33(4), 489-501.

Gonzalez-Espinosa, S., Ibáñez, S. J., Feu, S., \& Galatti, L. R. (2017). Programas de intervención para la enseñanza deportiva en el contexto escolar, PETB y PEAB. Retos, (31), 103-106.

González Villora, S., García López, L. M., Vicedo, P., Carlos, J., \& Contreras Jordán, O. R. (2011). Conocimiento táctico y la toma de decisiones en jóvenes jugadores de fútbol (10 años). Revista de Psicología del Deporte, 20, 79-97.

Gréhaigne, J.F., \& Godbout, P. (1995). Tactical knowledge in team sports from a constructivist and cognitivist perspective. Quest, 47(4), 490-505.

Griffin, L. L., Mitchell, S. A., \& Oslin, J. L. (1997). Teaching sports concepts and skills: A tactical games approach. Leeds: Human Kinetics .

Gutiérrez-Díaz, D., González-Víllora, S., García-López,L., \& Mitchell, S. (2011). Differences in decision-making between experienced and inexperienced invasion games players. Perceptual and Motor Skills, 112(3), 871-888.

Harvey, S., \& Jarrett, K. (2014).Areview of the game-centred approaches to teaching and coaching literature since 2006. Physical Education and Sport Pedagogy, 19(3), 278-300.

Kugler, P., Kelso, J. S., \& Turvey, M. (1982). On the control and coordination of naturally developing systems. The Development of Movement Control and Coordination, 5, 78.

Launder, A. G (2001). Play practice: The games approach to teaching and coaching sports. Champaign: Human Kinetics.

Mason, P. H. (2010). Degeneracy at multiple levels of complexity. Biological Theory, 5(3), 277-288.

Memmert, D. (2010). Game test situations: assessment of game creativity in ecological valid situations. International Journal of Sport Psychology, 41(4), 94.

Memmert, D., \& Roth, K. (2007). The effects of non-specific and specific concepts on tactical creativity in team ball sports. Journal 
of Sports Sciences, 25(12), 1423-1432.

Mitchell, S. A., Oslin, J. L., \& Griffin, L. L. (2003). Sport foundations for elementary physical education: A tactical games approach. Champaign: Human Kinetics.

Muñoz, D., Courel, J., Sánchez-Alcaraz, B.J., Díaz, J., Grijota, F.J., \& Muñoz, J.(2017). Análisis del uso y eficacia del globo para recuperar la red en función del contexto de juego en pádel. Retos, (31), 1922.

Newell, K. M. (1986). Constraints on the development of coordination. Motor development in children: Aspects of Coordination and Control, 34, 341-360.

Passos, P., Araújo, D., Davids, K., \& Shuttleworth, R. (2008). Manipulating constraints to train decision making in rugby union. International Journal of Sports Science and Coaching, 3(1), 125140.

Passos, P.L., \& Davids, K. (2015). Learning design to facilitate interactive behaviours in Team Sports. RICYDE. Revista Internacional de Ciencias del Deporte, 11(39), 18-32.

Pinder, R. A., Davids, K. W., Renshaw, I., \& Araújo, D. (2011). Representative learning design and functionality of research and practice in sport. Journal of Sport and Exercise Psychology, 33(1), 146-155.

Raab, M. (2007). Think SMART, not hard—a review of teaching decision making in sport from an ecological rationality perspective. Physical Education and Sport Pedagogy, 12(1), 1-22.

Renshaw, I., Chow, J. Y., Davids, K., \& Hammond, J. (2010). A constraints-led perspective to understanding skill acquisition and game play: A basis for integration of motor learning theory and physical education praxis? Physical Education and Sport Pedagogy, 15(2), 117-137.

Renshaw, I., Davids, K. W., Shuttleworth, R., \& Chow, J. Y. (2009). Insights from ecological psychology and dynamical systems theory can underpin a philosophy of coaching. International Journal of Sport Psychology, 40(4), 540-602.

Sanchez-Mora, D., Garcia Lopez, L. M., Del Valle Diaz, M. S., \& Solera Martinez, I. (2011). Spanish primary school students' knowledge of invasion games. Physical Education and Sport Pedagogy, 16(3), 251-264.

Seifert, L., Button, C., \& Davids, K. (2013). Key properties of expert movement systems in sport. Sports Medicine, 43(3), 167-178.
Seifert, L., Komar, J., Barbosa, T., Toussaint, H., Millet, G., \& Davids, K. (2014). Coordination Pattern Variability Provides Functional Adaptations to Constraints in Swimming Performance. Sports Medicine, 1333-1345.

Serra-Olivares, J., García-López, L. M., \& Sánchez-Mora, D. (2011). El juego modificado, recurso metodológico en el fútbol de iniciación. Retos: Nuevas Tendencias en Educación Física, Deporte y Recreación, (20), 37-42.

Serra-Olivares, J., Gonzalez-Víllora, S., \& García-López, L. M. (2011). Comparación del rendimiento de juego de jugadores de fútbol de 89 años en dos juegos modificados 3 contra 3. Cuadernos de Psicología del Deporte, 11(2), 77-92.

Serra-Olivares, J., González-Villora, S., García-López, L. M., \& Araújo, D. (2015). Game-Based Approaches’ Pedagogical Principles: Exploring Task Constraints in Youth Soccer. Journal of Human Kinetics, 46(1), 251-261.

Stolz, S., \& Pill, S. (2014). Teaching games and sport for understanding:Exploring and reconsidering its relevance in physical education. European Physical Education Review , 20(1), 36-71.

Tan, C. W. K., Chow, J. Y., \& Davids, K. (2012). 'How does TGfU work?’: examining therelationship between learning design in TGfU and a nonlinear pedagogy. Physical Education and Sport Pedagogy, 17(4), 331-348.

Thorpe, R., \& Bunker, D. (1997). A changing focus in games teaching. Physical Education in Schools, 2.

Thorpe, R., Bunker, D., \& Almond, L. (1986). Rethinking games teaching: Department of Physical Education and Sports Science, University of Technology.

Travassos, B., Duarte, R., Vilar, L., Davids, K., \& Araújo, D. (2012). Practice task design in team sports: Representativeness enhanced by increasing opportunities for action. Journal of Sports Sciences, 30(13), 1447-1454.

Travassos, B., Gonçalves, B., Marcelino, R., Monteiro, R., \& Sampaio, J. (2014). How perceiving additional targets modifies teams' tactical behavior during football small-sided games. Human Movement Science, 38, 241-250.

Vickers, J. N. (2007). Perception, cognition, and decision training: The quiet eye in action: Human Kinetics.

Whitacre, J., \& Bender, A. (2010). Degeneracy: a design principle for achieving robustness and evolvability. Journal of Theoretical Biology, 263(1), 143-153.

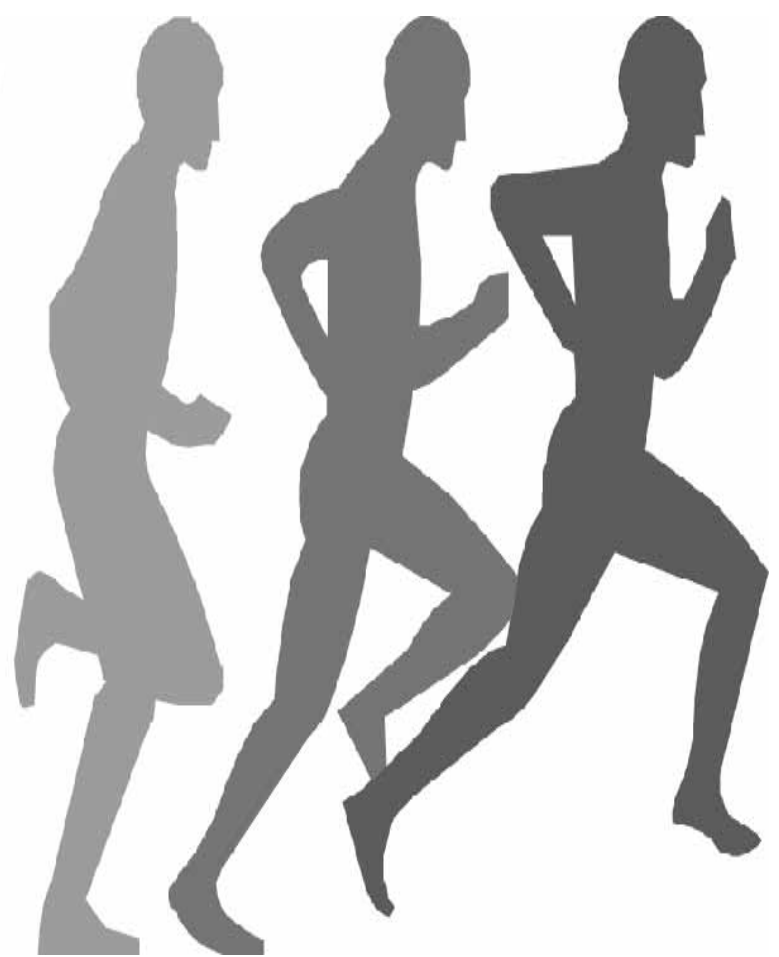

